\title{
Posttraumatische Fehlstellungen an der oberen Extremität im Kindesalter
}

Katharina Mörs, Johannes Frank, Britta Sundermann, Ingo Marzi

\section{Einleitung}

Die Behandlung von Verletzungen im Kindesalter hat sich in den letzten Jahren erheblich verbessert. Durch neue Operationsverfahren und Implantate wie die elastisch stabile intramedulläre Nagelung (ESIN), kanülierte Schrauben und an Kinder und Jugendliche adaptierte Nagel- und Plattensysteme ist heutzutage eine fugenschonende Reposition und Stabilisierung von Frakturen im Kindesalter möglich [1,2].

Gerade an der oberen Extremität ist aufgrund des geringen Wachstumspotenzials im Bereich des Ellenbogengelenkes eine exakte Reposition und Stabilisierung essenziell, um ein Fehlwachstum zu vermeiden. Dennoch kommt es immer wieder durch das initiale Trauma (direkte Verletzung der Fuge), aber auch durch die operative und konservative Behandlung der typischen kindlichen Verletzungen zu Wachstumsstörungen, die teils erhebliche funktionelle Auswirkungen haben können. In einigen Fällen macht dies eine sekundäre Rekonstruktion unumgänglich, um die Funktion wiederherzustellen. Die Indikation zur Korrekturosteotomie sollte sich im Wesentlichen an den funktionellen Einschränkungen orientieren.

Bei fehlverheilten suprakondylären Humerusfrakturen mit Varusdeformität, Innenrotationsfehler und Antekurvation empfiehlt sich bei Kleinkindern nach sorgfältiger präoperativer Planung die suprakondyläre Korrekturosteotomie mit Fixateur externe. Bei Jugendlichen kann auch eine Korrekturosteotomie und Stabilisierung mittels Plattenosteosynthese erfolgen.

Fehlverheilte Monteggia-Verletzungen sollten möglichst frühzeitig durch Korrekturosteotomien, meist im Bereich der Elle, mit Einstellung des Radiuskopfes behandelt werden und haben eine gute Prognose im Gegensatz zu chronischen Luxationsstellungen mit Ausbildung sekundärer Deformitäten.

Einschränkungen der Unterarmumwendbeweglichkeit nach in Fehlstellung verheilten Unterarmschaftfrakturen können in den meisten Fällen durch Korrekturosteotomien und intramedulläre Nagelung gut therapiert werden.
Am distalen Unterarm kann es im Rahmen der konservativen Behandlung in Einzelfällen zu Funktionseinschränkungen im Bereich des distalen Radioulnargelenkes (DRUG) und der Handgelenksbeweglichkeit kommen. Auch bei Fugenverletzungen kann es zu erheblichen Störungen des Wachstums kommen, wie bspw. einem vorzeitigen Fugenschluss. Hier kann die Verlängerung des Radius notwendig werden, um die Kongruenz im DRUG wiederherzustellen.

\section{Indikationsstellung zur Umstellungsosteotomie}

Die Anzahl notwendiger sekundärer Rekonstruktionen im Bereich der oberen Extremität nach Verletzung im Kindesalter ist bei optimierter primärer Therapie selten und die Indikation in jedem Fall differenziert und individuell zu stellen [2].

Die Korrekturoperation wird häufig von den Eltern und den Kindern aufgrund kosmetischer Einschränkungen gewünscht. Hierzu zählen bspw. der Cubitus varus oder Cubitus valgus nach suprakondylären Humerusfrakturen oder Condylus-radialis-Frakturen, aber auch endgradige Bewegungseinschränkungen oder Überstreckungen. In diesen Fällen müssen die Eltern und das Kind ausführlich darüber beraten werden, dass aus allein kosmetischer Indikation im Kindesalter üblicherweise keine operative Korrektur notwendig ist.

Problematisch ist dabei die Abschätzung von Spätfolgen und der Wahrscheinlichkeit, dass diese auftreten. Zum Beispiel die Irritation des N. ulnaris beim Cubitus valgus. Bei Jugendlichen oder jungen Erwachsenen kann nach entsprechender umfassender Aufklärung eine Korrektur aus kosmetischen Gründen vorgenommen werden, was glücklicherweise sehr selten erfolgt.

Indikationen zur frühzeitigen Umstellungsosteotomie sind allerdings funktionelle Beeinträchtigungen, wie z. B. die aufgehobene oder eingeschränkte Drehung nach Unterarmschaftfrakturen, darüber hinaus erhebliche Achsabweichungen im Bereich des Ellenbogengelenkes deutlich $>10^{\circ}$ Varus- und Valgusfehlstellung und vor allem Beuge- und Streckdefizite nach fehlverheilten Monteggia-Verletzungen, welche die Greiffunktion erheblich be- 
- Tab. 1 Wesentliche Indikationen zur Korrekturosteotomie an der oberen Extremität.

\begin{tabular}{|l|l|}
\hline Beeinträchtigung/Störung & Auswirkung/Ursache \\
\hline $\begin{array}{l}\text { eingeschränkte Ellenbogenbeugung } \\
\text { fehlende oder erheblich eingeschränkte Pronation oder Supination }\end{array}$ & $\begin{array}{l}\text { Hand kommt nicht an Mund und Hinterkopf } \\
\text { Stände fallen aus der Hand }\end{array}$ \\
\hline Nervenirritationen & N.-ulnaris-Dehnung bei Cubitus valgus \\
\hline erheblich eingeschränkte Handgelenksbeweglichkeit & bei Verkürzung des Radius oder Fehlstellung im DRUG \\
\hline ausgeprägte kosmetische Störungen & \\
\hline
\end{tabular}

- Tab. 2 Posttraumatischen Fehlstellungen im Bereich des distalen Humerus.

\begin{tabular}{|l|l|l|}
\hline Verletzung & Deformität & Ursache \\
\hline suprakondyläre Humerusfrakturen & Cubitus varus & ulnarer Fugenschluss, radiale Stimulation \\
\hline Condylus-radialis-Fraktur & Cubitus varus & radiale Stimulation \\
\hline Condylus-radialis-Fraktur & Cubitus valgus & radiale Pseudarthrose, ulnare Stimulation \\
\hline $\begin{array}{l}\text { Condylus-radialis-Fraktur } \\
\text { Epicondylus-ulnaris-Fraktur }\end{array}$ & Verplumpung des Ellenbogengelenkes & Pseudarthrose, nicht refixierter ulnarer Epicondylusabriss \\
\hline Y-Fraktur distaler Humerus & Fischschwanzdeformität & \\
\hline & & zentraler Verschluss der Fuge mit Fehlwachstum \\
\hline
\end{tabular}

einträchtigen. Übersehene Monteggia-Verletzungen haben den größten Anteil an Korrekturoperationen im Bereich der oberen Extremitäten, da es hier häufig zu erheblichen Einschränkungen der Beugung kommen kann [46]. Hingegen sind Verlängerungsosteotomien bei vorzeitigem Fugenschluss nach distaler Epiphysenverletzung des Radius oder suprakondyläre Umstellungen nach suprakondylären Humerusfrakturen seltener.

Bei der Indikationsstellung sowie der Beratung vor einer Korrekturoperation werden die in $>$ Tab. 1 angegebenen Parameter zur Hilfe genommen. Es handelt sich hier im Wesentlichen um Veränderungen der Ellenbogenachse mit funktioneller Einschränkung der Beweglichkeit, Rotationseinschränkungen des Unterarms nach Unterarmschaftfrakturen und Verkürzungen des Radius bei vorzeitigem Fugenschluss mit Inkongruenz im DRUG.

Im Folgenden werden beispielhaft die folgenden Umstellungsosteotomien an der oberen Extremität dargestellt:

- posttraumatische Deformitäten des distalen Humerus

- posttraumatische Bewegungseinschränkung nach Monteggia-Verletzung

- posttraumatische Einschränkung der Unterarmrotation nach Unterarmschaftfraktur

- posttraumatische Inkongruenz im distalen Radioulnargelenk

\section{Posttraumatische Fehlstellungen des distalen Humerus}

Am distalen Humerus finden sich die in $>$ Tab. 2 aufgelisteten posttraumatischen Fehlstellungen.

Bei fehlverheilten suprakondylären Humerusfrakturen entsteht am ehesten eine Varusdeformität mit gleichzeitiger Innenrotation und Antekurvation durch die bei dem Frakturmechanismus erlittene Fehlstellung und unzureichende Reposition und/oder Stabilisierung. Bei dieser Varusdeformität zeigt sich nicht immer eine Bewegungseinschränkung. Sollten die Bewegungseinschränkungen deutlich stören oder im Alter zunehmen, ist eine operative Korrektur möglich. Hier ist einerseits die operative Korrekturosteotomie mit Plattenosteosynthese bei vollendetem Fugenschluss möglich. - Abb. 1 zeigt den Verlauf eines Cubitus varus mit deutlicher Beugeeinschränkung von $30^{\circ}$ bei einem 15-jährigen Mädchen; die Korrektur erfolgte hier mittels Plattenosteosynthese ( $\bullet$ Abb. 1).

Im Kindesalter wird häufig nach entsprechender operativer Planung die suprakondyläre Korrekturosteotomie mit Fixateur externe durchgeführt [7]. Dabei kann der Fixateur externe von radial angebracht werden, um die Achse zu korrigieren. Bei dieser Operation ist die genaue präoperative Planung der Korrekturosteotomie hilfreich, um ein gutes Korrekturergebnis zu erreichen. Die stabilen modularen Verbindungen des Fixateur externe erlauben 

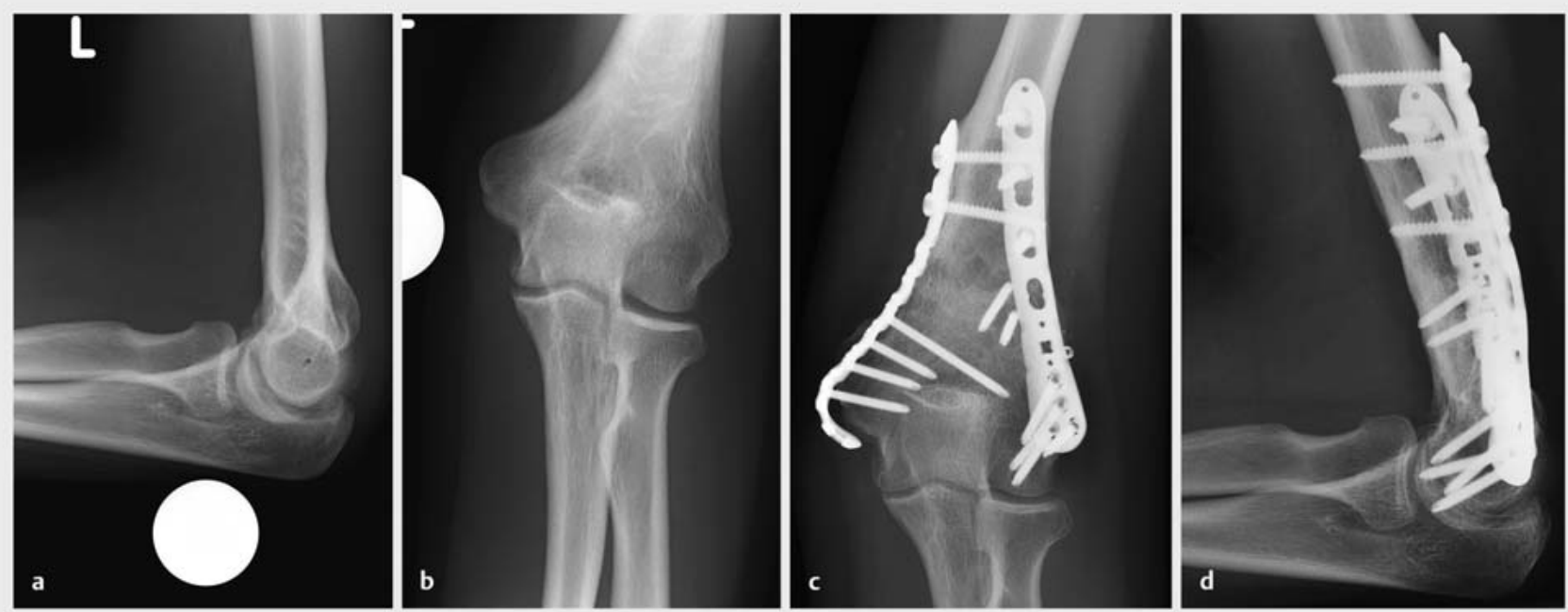

- Abb. 1 Röntgenbilder einer fehlverheilten suprakondylären Humerusfraktur mit Cubitus varus und Beugedefizit bei einem 15-jährigen Mädchen. a, b Ausgangsbilder. c, d Ausheilung nach Korrekturosteotomie mit Plattenosteosynthese, Revision und Spongiosaplastik.

eine funktionelle Nachbehandlung. Ein solcher Fall aus unserer Klinik ist in Frank et al. in den dortigen Abb. 6 und 7 dargestellt [3].

Die Condylus-radialis-Fraktur ist eine schwierig zu diagnostizierende Fraktur, die aufgrund des Muskelzuges zu erheblicher Dislokation neigen kann. Daher ist hier selbst bei initial anatomischer Stellung eine gipsfreie Röntgenkontrolle nach 4-5 Tagen unbedingt erforderlich. Sollte es zu einer Dislokation kommen, handelt es sich um eine instabile Fraktur, die operativ stabilisiert werden muss [2, 8, 9]. Im Rahmen dieser operativen Stabilisierung erfolgt einerseits die offene Einstellung der Gelenkfläche und Fixation mit Kirschner-Draht oder Schraube sowie des metaphysären Fragmentes bevorzugt bei ausreichender Größe mittels Zugschraubenosteosynthese von dorsoradial $[2,9]$. Wird eine Condylus-radialis-Fraktur nicht unmittelbar korrekt versorgt, kann hieraus eine Pseudarthrose mit entsprechender Cubitus-valgus-Fehlstellung entstehen, die meist einer operativen Korrektur bedarf. Aus der Valgusdeformität resultiert durch die Fehlstellung neben der störenden Kosmetik häufig eine Irritation des N. ulnaris, da dieser überdehnt wird. Besonders problematisch bei der Korrektur ist eine Funktionseinschränkung, die aus der Gelenkbeteiligung dieser Fraktur mit intraartikulärer Deformität resultiert [3].

\section{Bewegungseinschränkungen nach fehlverheilter oder übersehener Monteggia-Verletzung}

Einschränkungen der Unterarmbeweglichkeit, insbesondere der Beugung im Ellenbogen bis max. 90 mögliche Folge fehlverheilter oder übersehener Monteggia-Verletzungen.

Monteggia-Läsionen gehören zu den am häufigsten übersehenen Verletzungen, da oft eine Biegungsverletzung der Ulna im Sinne einer Bowing-Fraktur vorliegt, sodass man keine eigentliche Fraktur der Ulna sieht. Daher muss unbedingt darauf geachtet werden, dass der Radiuskopf sich in allen Ebenen auf die Mitte des Capitulums projiziert ( $\triangleright$ Abb. 2).

Um eine Monteggia-Verletzung und akute Luxation auszuschließen, muss daher bei jeder Unterarmfraktur und Ellenbogenverletzung auf Folgendes geachtet werden: Der Unterarm muss vollständig in 2 Ebenen geröntgt sein. Auf jedem Bild des Ellenbogens muss sich die Schaftlinie des Radiuskopfes auf den Capitulumkern zentrieren. Vergleichsaufnahmen mit der Gegenseite sind bei $V$.a. chronische Luxation mit sekundären Deformitäten, dem V.a. kongenitale bzw. entwicklungsbedingte Fehlstellungen und ohne adäquates Trauma sinnvoll.

Die Monteggia-Verletzungen werden nach Bado klassifiziert ( $\triangleright$ Abb. 3 ).

Bei der Monteggia-Verletzung werden am häufigsten ulnare Biegungsbrüche und Grünholzfrakturen Typ A und 
B übersehen, die zu einer Dislokation des Radiusköpfchens führen.

Bei den dorsalen Luxationen und den Luxationen Typ D und E muss vor allem bei Verplumpung und Veränderung des Radiuskopfes auch an chronische oder kongenitale Deformität gedacht werden, die von akuten MonteggiaLäsionen abgegrenzt werden müssen. Bei der chronischen Luxation sind die Ergebnisse einer operativen Behandlung ausgesprochen limitiert, weshalb hier die Indikation sehr differenziert und nach umfassender Aufklärung gestellt werden sollte. Besondere Kennzeichen einer chronischen Luxation sind Deformitäten des Radiuskopfes, der aufgrund chronischer Umbauvorgänge meist eine Achsabweichung zeigt. Zusätzlich zeigen sich oft Umformungen der proximalen Elle im Bereich des proximalen Radioulnargelenkes (PRUG), sodass der Formschluss fehlt und der Radiuskopf meist nach ventral luxiert verbleibt [4].

Die Indikation zur Revision ist bei praktisch allen fehlverheilten Monteggia-Verletzungen gegeben, wobei unbedingt eine frühzeitige Revision innerhalb der ersten 6 Wochen bis zu 3 Monaten empfohlen wird, um ein besseres Ergebnis zu erzielen.

Das Prinzip der Rekonstruktion besteht in einer Korrekturosteotomie der Ulna mit Angulation und bei Bedarf Distraktion. In diesem Fall kommt es dann durch den Längengewinn und die Angulation der Ulna zu einem Wiedereinrichten des Radiusköpfchens im Ellenbogengelenk. Die Korrektur kann bei einer frühzeitigen Rekonstruktion einzeitig erfolgen. Bei einer späten Rekonstruktion ist zuerst ein Längengewinn erforderlich, um dann in einem 2. Eingriff die Reposition des Radiuskopfes durchführen zu können. Anschließend muss der Fixateur externe noch einige Wochen bis zur knöchernen Konsolidierung der Ulna belassen werden, oder aber auch ein Verfahrenswechsel auf eine Platte erfolgen, sodass letztlich meistens 4 operative Eingriffe erforderlich werden. Im Verlauf muss dann nicht nur der Ellenbogen kontrolliert werden,

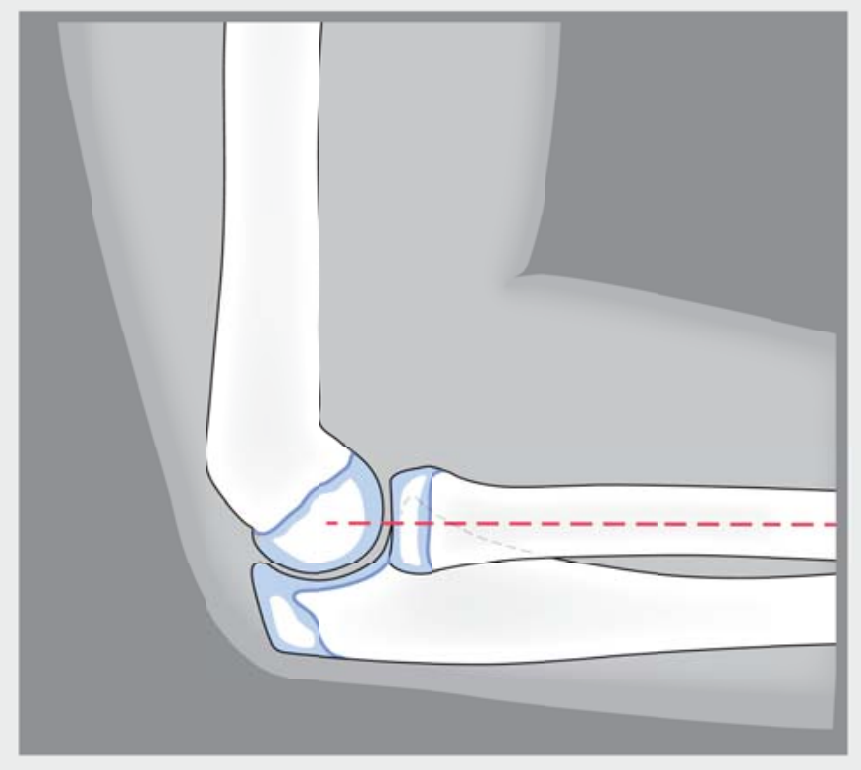

- Abb. 2 Die Achse des Radius muss sich nach proximal durch den Radiuskopf immer auf das Capitulum humeri radialis projizieren (StoerenLinie).

sondern auch das Handgelenk. Inkongruenzen können dort nach Konsolidierung des Ellenbogens Nivellierungsoperationen erforderlich machen (z. B. Ellenverkürzung), meist nach Ablauf eines Jahres oder mehr.

Am Beispiel in $\mathbf{A b b . 4}$ erkennt man die übersehene Monteggia-Verletzung mit Ventralluxation des Radiuskopfes und der Verbiegung der Ulna. Nach der Korrekturoperation stellt sich das Gelenk wieder regelrecht ein. Im Wesentlichen werden die Korrekturoperationen zunächst meist mittels Fixateur externe durchgeführt, da man mit diesem die Ulna so angulieren kann, dass sich der Radiuskopf einstellt. Außerdem kann man hier einen Distraktor integrieren, der den Längengewinn ermöglicht, damit der Radiuskopf aus der proximalen Position in Relation

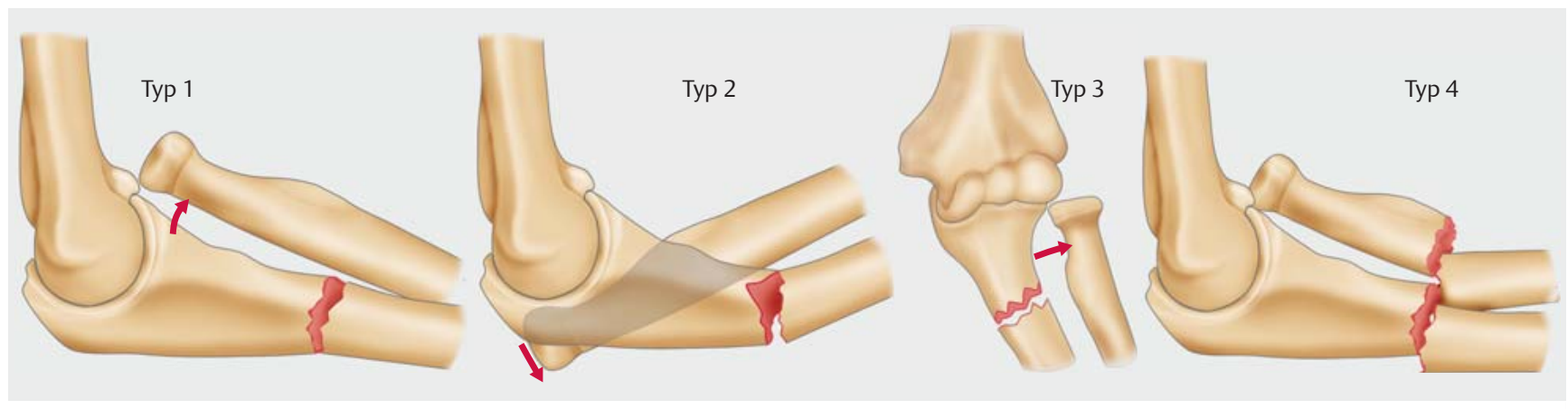

- Abb. 3 Bado-Klassifikation der Monteggia-Verletzungen im Kindesalter. Quelle: Meffert R. Proximale Ulnafraktur. In: Müller L, Hollinger B, Burkhart K, Hrsg. Expertise Ellenbogen. Stuttgart: Thieme; 2016. 

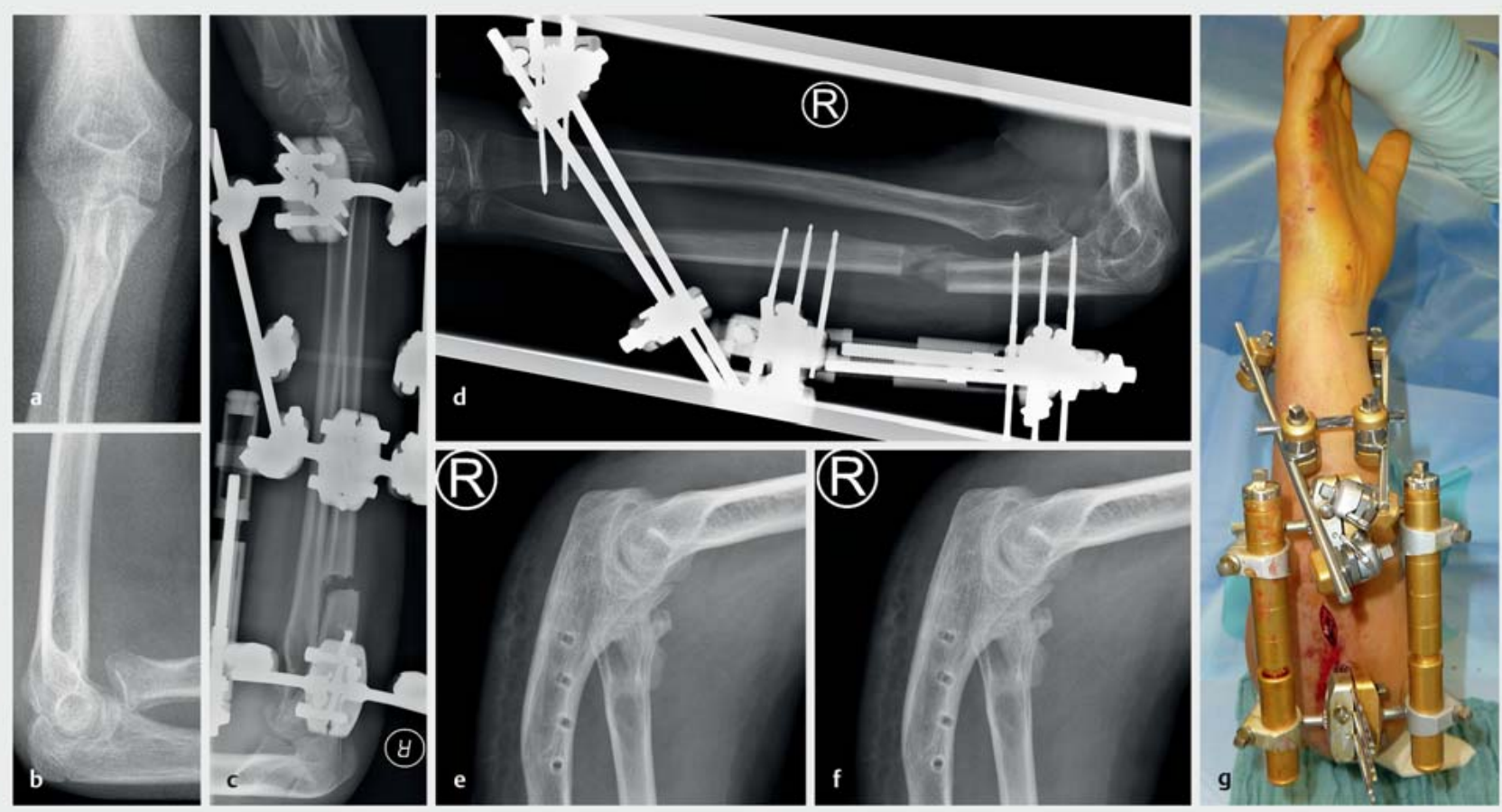

- Abb. 4 Fall eines 12-jährigen Mädchens mit initial übersehener Monteggia-Verletzung mit Ulna-Bowing und in der Folge ventraler Radiusköpfchenluxation. a, b Ventrale Radiusköpfchenluxation nach Ausheilung der Fraktur. c Anlage des Distraktionsfixateurs nach Korrekturosteotomie der Ulna. d Distraktion der Ulna mit Kallusbildung. e Konsolidierung mit Platte. f Korrekte Stellung bei leichter Deformation nach Metallentfernung.

zum Capitulum humeri radialis herausgebracht werden kann. Bei einer Verlängerung nach einer chronischen Luxation muss mit dem Fixateur auch der Radius gefasst werden, da es sonst zu einer Verschiebung im Bereich der Membrana interossea und des DRUG kommt mit erheblichem Ellenvorschub und konsekutiver Beeinträchtigungen der Handgelenkfunktion und Unterarmdrehung.

Bei einer frühen Revision kann eine Monteggia-Verletzung auch mithilfe eines ESIN-Drahtes oder mit einer kurzen Platte korrigiert werden [2].

Insgesamt sind die Ergebnisse bei früher Korrektur von Monteggia-Verletzungen gut. Bei später Korrektur sind sie deutlich schlechter, da nur Teilziele erreicht werden können, was durch die sekundären Veränderungen der Knochen- und Gelenkstrukturen bedingt ist. Zudem sind dann komplexe langwierige Behandlungen erforderlich. Meist hat sich dann der Radiuskopf bereits verformt und der Raum für den Radiuskopf neben der Ulna, gegenüber dem Capitulum humeri, ist eingeengt und deformiert, was die Einstellung des Gelenkes erheblich erschwert.

Bei einer aussichtslosen Situation mit persistierender Beschwerdesymptomatik kann man im Adoleszentenoder Erwachsenenalter den Radiuskopf resezieren und den Schaft mit einer Anconeusmuffe aus dem M. anconeus, im Sinne einer Arthroplastik, abdecken
[11]. Hiermit lassen sich zufriedenstellende Ergebnisse erreichen.

\section{Einschränkung der Unterarmrotation bei fehlverheilten Unterarmschaftfrakturen}

Unterarmschaftfrakturen sind eine der häufigsten Verletzungen im Kindesalter. Sie sind in aller Regel deutlich disloziert, was auf den Muskelzug einerseits proximal durch den M. pronator teres und den M. supinator und andererseits distal durch den M. pronator quadratus zurückzuführen ist. Unterarmschaftfrakturen können als vollständige Unterarmschaftfrakturen, als Biegungsbrüche oder als Grünholzbrüche auftreten. Es können auch Kombinationen einer vollständigen Fraktur bspw. des Radius und eines Biegungsbruches der Ulna auftreten $[2,8]$.

Bei Unterarmschaftfrakturen hat sich die operative Behandlung weitestgehend durchgesetzt $[2,8]$. Bis auf Kinder im Alter von 2-3 Jahren wird bei Schaftfrakturen oder Grünholzfrakturen regelhaft das Überbrechen der Fraktur und die Stabilisierung mittels elastisch stabiler intramedullärer Nagelung durchgeführt $[1,2,12]$. Dies hat zu einer erheblichen Verbesserung der Beweglichkeit durch die Möglichkeit einer frühfunktionellen Behandlung geführt [2,13]. 


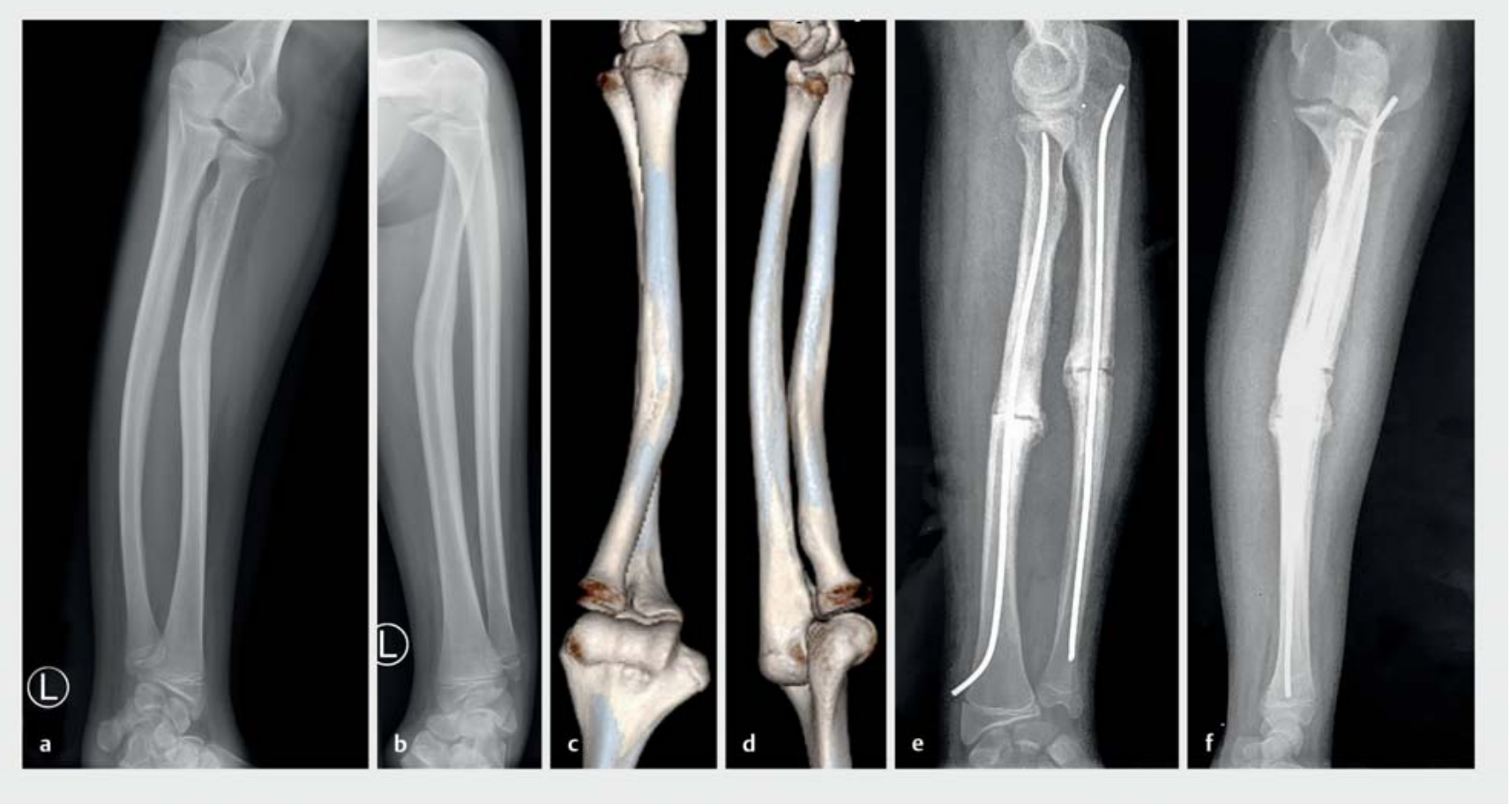

- Abb. 5 Röntgenbilder einer fehlverheilten Unterarmschaftfraktur nach konservativer Behandlung bei einem 14-jährigen Jungen. a, b Ausgangsbilder. c, d 3-D-Low-Dose-Array-CT. e, $f$ Zunehmende Konsolidierung bei freier Funktion nach 5 Monaten.

Bei Kindern unter 2-3 Jahren kann bei wenig dislozierten Frakturen auch die konservative Behandlung durchgeführt werden. Die klassische Behandlung mittels Gipskeilung hat dabei in unseren Breiten praktisch keinen Stellenwert mehr. Vor allem bei Grünholzfrakturen oder vollständigen Frakturen auf gleicher Höhe kommt es bei konservativer Therapie immer wieder zu Bewegungseinschränkungen und Funktionsstörungen, die einer operativen Korrektur bedürfen [14]. Zu berücksichtigen ist insbesondere, dass sich bei Grünholzfrakturen unter konservativer Behandlung die Fehlstellung erheblich verstärken kann [13].

Ab einer Dislokation von $>10^{\circ}$ ist generell mit dem Auftreten von Störungen der Umwendbewegung zu rechnen, wobei diese oft erst ab einem höheren Fehlstellunggrad vorkommen. Andererseits gibt es auch Fälle, in denen durch die Kombination von Fehlstellungen Störungen der Umwendbewegung entstehen, die kaum zu erkennen sind. Um solche Veränderungen darzustellen, verwenden wir ein Low-Dose-CT mit 3-D-Rekonstruktion im Seitenvergleich ( $\nabla$ Abb.5c,d). Die Einschränkungen der Umwendbewegung sind meist rein mechanisch bedingt durch die Verbiegung und in der Folge gestörte Drehung des Radius um die Ulna. Des Weiteren kommt es bei Unterarmschaftfrakturen auf gleicher Höhe bei konservativer Behandlung gelegentlich zu Brückenbildungen oder Synostosen, welche die Unterarmdrehbeweglichkeit beeinträchtigen.
Einschränkungen der Umwendbewegungen infolge von Unterarmfrakturen betreffen meistens die Supination, teilweise aber auch die Supination und Pronation. Dies führt zu erheblichen Beeinträchtigungen der Kinder auch bei der späteren Berufswahl. Daher ist in solchen Fällen die Indikation zur Korrekturosteotomie bereits frühzeitig gegeben.

- Abb. 5 zeigt den typischen Fall einer fehlverheilten Unterarmfraktur bei einem 14-jährigen Jungen mit einer erheblichen Rotationseinschränkung mit einer Supination bis $10^{\circ}$ und einer Pronation bis $90^{\circ}$. Aufgrund der Beschwerdesymptomatik erfolgte hier die Korrekturosteotomie von Ulna und Radius durch lokale Inzisionen über dem Hauptdrehpunkt. Anschließend wurde die ESINNagelung von Radius und Ulna außerhalb der Wachstumsfugen mittels ESIN-Drähten der Stärke $2,5 \mathrm{~mm}$ durchgeführt. Die Nagelung des Radius erfolgte klassischerweise retrograd unter Schonung des R. superficialis des N. radialis, die der Ulna antegrad vom Olecranon ausgehend. Die ESIN-Drähte werden so eingebracht, dass sie in Neutralstellung fixiert sind. Intraoperativ muss abschließend geprüft werden, ob eine vollständige Supination und Pronation möglich ist. Nach einer kurzzeitigen Ruhigstellung in einer Oberarmgipsschiene für wenige Tage kann eine funktionelle Nachbehandlung durchgeführt werden. Dieses Vorgehen führt üblicherweise zu guten Ergebnissen, wie auch im genannten Fallbeispiel. 
Nach Ausheilung konnte eine freie Umwendbeweglichkeit erreicht werden.

\section{Fehlstellungen am distalen Unterarm und im Bereich des distalen Radioulnargelenkes}

Bei kindlichen Verletzungen im Bereich des distalen Unterarms steht die konservative Behandlung im Vordergrund [15-17]. Lediglich bei komplett dislozierten Frakturen oder Dislokation der Gelenkfläche $>20-30^{\circ}$ ist je nach Alter des Kindes eine primäre operative Intervention und Reposition nach Kapandji oder mit Kirschner-Drähten erforderlich. Bei Adoleszenten ist bei Frakturen am meta-/diaphysären Übergang eine palmare Plattenosteosynthese zu erwägen, die aber die Fuge schont.

Indikationen für Umstellungsosteotomien sind fehlverheilte Galeazzi-Verletzungen oder distale Ulna- oder Radiusfrakturen, die zu einer Inkongruenz im DRUG und somit zu einer Störung der Drehung des Unterarms führen. Eine weitere Verletzungsgruppe, bei der eine Umstellungsosteotomie notwendig werden kann, sind die diametaphysären Frakturen des Unterarms, die gerade bei Kindern ab 10 Jahren bei einer Fehlstellung $>10^{\circ}$ nicht mehr achsgerecht ausheilen. Insbesondere bei einer Grünholzfraktur kann sich die Fehlstellung noch verstärken.

Häufige Indikationen zur operativen Korrektur nach distalen Unterarmfrakturen sind daher Fehlstellungen, die nach Grünholz- oder Biegungsfrakturen entstehen und letztlich die Handgelenksbeweglichkeit erheblich beeinträchtigen oder Schmerzen im Bereich des DRUG verursachen. In diesen Fällen ist dann häufig eine Korrekturosteotomie mit konsekutiver Plattenosteosynthese indiziert. Hierbei muss differenziert werden, ob eine Verlängerung des Radius oder eine Verkürzung der Ulna die bessere Lösung ist [2].

Die Korrektur und Verlängerung des Radius kann über einen palmaren Zugang mit einer winkelstabilen Platte erfolgen. Die Verkürzungsosteotomie der Ulna ist am günstigsten bei Wachstumsabschluss und wird mit einer standardisierten Platte durchgeführt. Bei einer ausgeprägten Längendiskrepanz kann zunächst eine Radiusverlängerung mittels Distraktionsosteogenese notwendig werden.

\section{Schlussfolgerungen}

Die Behandlung von Frakturen im Kindesalter hat sich durch die Einführung adaptierter Operationsverfahren, vor allem der ESIN, der kanülierten Schrauben und fugenschonenden Techniken erheblich verbessert. Bei korrekter Indikationsstellung zur operativen bzw. konservativen
Therapie unter Anwendung der empirisch bekannten Kriterien und Grenzwerte kommt es selten zu korrekturbedürftigen Fehlstellungen. Bei posttraumatischen Fehlstellungen ist die Indikation zur operativen Korrektur im Wesentlichen nach funktionellen Gesichtspunkten zu stellen. Kosmetische Aspekte sollten, wenn überhaupt, erst bei Adoleszenten diskutiert werden. Der ideale Zeitpunkt für eine Korrekturosteotomie ist nicht endgültig geklärt. Lange Zeit herrschte die Meinung vor, dass eine solche erst nach Wachstumsabschluss erfolgen sollte. Derzeit geht man jedoch davon aus, dass bei einer störenden und relevanten Funktionseinschränkung, bspw. einer Einschränkung der Umwendbewegung des Unterarms, die Korrektur möglichst frühzeitig durchgeführt werden sollte, um eine gute Weiterentwicklung zu ermöglichen. Gerade bei Gelenkbeteiligung muss eine frühzeitige Korrektur durchgeführt werden, da sekundäre Umformungen, die sich nach der primären posttraumatischen Deformität entwickeln, eine Korrektur erschweren oder gar unmöglich machen.

Fehlstellungen, die zu einer erheblichen Bewegungseinschränkung im Bereich der oberen Extremität führen, finden sich insbesondere im Bereich des Ellenbogens sowie des Unterarms, aber auch im Bereich des distalen Radioulnargelenkes und des Handgelenkes.

\section{Interessenkonflikt}

Die Autoren versichern, dass keine Interessenkonflikte vorliegen.

\section{Autorinnen/Autoren}

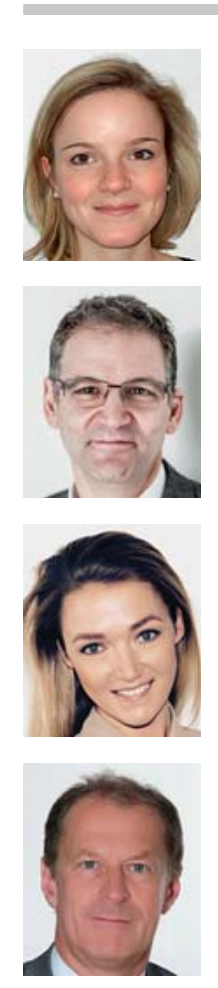

Katharina Mörs Hand- und Wiederherstellungschirurgie, Universitätsklinikum Frankfurt

Prof. Dr. med., Stellvertretender Direktor, Klinik für Unfall-, Hand- und Wiederherstellungschirurgie, Universitätsklinikum Frankfurt

\section{Britta Sundermann}

Dr. med., Assistenzärztin, Klinik für Unfall-, Hand- und Wiederherstellungschirurgie, Universitätsklinikum Frankfurt

\section{Ingo Marzi}

Prof. Dr. med., Direktor, Klinik für Unfall-, Handund Wiederherstellungschirurgie, Universitätsklinikum Frankfurt

\section{Johannes Frank}




\section{Korrespondenzadresse}

\section{Dr. med. Katharina Mörs}

Klinik für Unfall-, Hand- und Wiederherstellungschirurgie Universitätsklinikum Frankfurt

Theodor-Stern-Kai 7

60590 Frankfurt am Main

Tel.: 069/6301-6123

katharina.moers@kgu.de

Literatur

[1] Dietz HG. Elastic stable intramedullary Nailing (ESIN) in Children: 30 Step by Step Case Descriptions. Stuttgart; New York: Thieme; 2006

[2] Marzi I, Rose S. Kindertraumatologie. 2. Aufl. Springer; 2016

[3] Frank J, Sander AL, Voth M et al. Sekundäre Rekonstruktionen am Ellenbogengelenk im Kindesalter. Trauma Berufskrankh 2017; 19: 126-134

[4] Ulrich D, Voth M, Frank J et al. Luxationsfrakturen am Ellenbogengelenk im Kindesalter. Trauma Berufskrankh 2015; 17: 253-259

[5] Slongo T, Fernandez FF. Die fehlverheilte Monteggia-Verletzung im Kindes- und Jugendalter. Unfallchirurg 2011; 114: 311

[6] Francisco FF, Langendörfer M, Wirth T et al. Korrektur von veralteten Monteggia-Verletzungen im Kindes- und Jugendalter. Obere Extrem 2014; 9: 178-185

[7] Linhart WE, Kraus T. [Reconstruction of humeroradial joint]. Oper Orthop Traumatol 2008; 20: 396-408
[8] Schmittenbecher PP. Frakturen der oberen Extremität im Kindes-/Wachstumsalter. Chirurg 2017; 88: 451-466

[9] Fernandez FF, Eberhardt O, von Kalle T et al. Kirschner-DrahtOsteosynthese von Frakturen des Condylus radialis im Kindesund Jugendalter. Obere Extrem 2009; 4: 26-32

[10] Letts $\mathrm{M}$, Locht R, Wiens ]. Monteggia fracture-dislocations in children. J Bone Joint Surg Br 1985; 67-B: 724-727

[11] Hell AK, Von LL. Growth behaviour after fractures of the proximal radius: differences to the rest of the skeleton. Unfallchirurg 2014; 117: 1085-1091

[12] Jayakumar P, Jupiter JB. Reconstruction of malunited diaphyseal fractures of the forearm. Hand (N Y) 2014; 9: 265-273

[13] von Laer L, Kraus R, Linhart WE. Frakturen und Luxationen im Wachstumsalter. Stuttgart: Thieme; 2012

[14] Schmittenbecher PP, Dietz HG, Uhl S. Late results of forearm fractures in childhood. Unfallchirurg 1991; 94: 186-190

[15] Laurer H, Sander A, Wutzler S et al. [Therapy principles of distal fractures of the forearm in childhood]. Chirurg 2009; 80: $1042-1052$

[16] Lieber J, Sommerfeldt DW. Diametaphyseal forearm fracture in childhood. Pitfalls and recommendations for treatment. Unfallchirurg 2011; 114: 292-299

[17] Illian CH, Veigel B, Rixen D. Versorgungsmöglichkeiten kindlicher Frakturen. Trauma Berufskrankh 2012; 14: 232-238

Bibliografie

DOI https://doi.org/10.1055/a-0624-2511

OP-JOURNAL 2018; 34: 302-309 @ Georg Thieme Verlag KG Stuttgart · New York ISSN 0178-1715 